Revue internationale P.M.E.

Économie et gestion de la petite et moyenne entreprise

\title{
L'effort de recherche de la PME industrielle face au développement de produits nouveaux : la prépondérance des moyens humains
}

\section{Mohamed Bayad}

Volume 6, numéro 1, 1993

URI : https://id.erudit.org/iderudit/1008162ar

DOI : https://doi.org/10.7202/1008162ar

Aller au sommaire du numéro

Éditeur(s)

Presses de l’Université du Québec

ISSN

0776-5436 (imprimé)

1918-9699 (numérique)

Découvrir la revue

Citer cet article

Bayad, M. (1993). L'effort de recherche de la PME industrielle face au développement de produits nouveaux : la prépondérance des moyens humains. Revue internationale P.M.E., 6(1), 29-48.

https://doi.org/10.7202/1008162ar
Résumé de l'article

L'objet de cet article est de présenter les résultats d'une analyse exploratoire portant sur la relation entre l'effort de recherche de la petite entreprise et son activité innovatrice en produit. Basés sur les données du panel PMI Lorraines, nos résultats soulignent l'importance, au niveau de l'effort de recherche, de la dimension des moyens humains et de l'orientation client dans la discrimination des pratiques innovatrices dans les produits de la PMI. Par contre, la dimension financière, associée tant aux dépenses de recherche et développement qu'aux dépenses d'études de marché, ne semblent pas intervenir dans la différenciation des comportements d'innovation produit. 


\title{
L'effort de recherche de la PME industrielle face au développement de produits nouveaux : la prépondérance des moyens humains
}

\author{
Mohamed BAYAD* \\ Groupe ICN-Université Nancy II
}

\begin{abstract}
RÉSUMÉ
L'objet de cet article est de présenter les résultats d'une analyse exploratoire portant sur la relation entre l'effort de recherche de la petite entreprise et son activité innovatrice en produit. Basés sur les données du panel PMI Lorraines, nos résultats soulignent l'importance, au niveau de l'effort de recherche, de la dimension des moyens humains et de l'orientation client dans la discrimination des pratiques innovatrices dans les produits de la PMI. Par contre, la dimension financière, associée tant aux dépenses de recherche et développement qu'aux dépenses d'études de marché, ne semblent pas intervenir dans la différenciation des comportements d'innovation produit.
\end{abstract}

\section{ABSTRACT}

The goal of this paper is to present the results of a study on the relation in terms of product between the research efforts of a small company and its innovative activity. Based on data provided by the SMI Lorraine panel, our results underline the importance of the level of the research effort, of the human ressources dimension and of customer orientation in the differenciation of the innovative practices of the SMI. On the contrary, the financial dimension, associated with the costs of research and development as well as with market research expenses, dos not seem to intervene in the differenciation of innovative behaviour.

* Mohamed Bayad, docteur en économie industrielle, enseigne le management de la PME ainsi que les méthodes quantitatives appliquées à la gestion au sein du Groupe ICN (Institut commercial de Nancy, Université de Nancy II). Il est également responsable d'une équipe de recherche pour la PME (ERPME au sein d'ICN Recherche) dans le cadre de laquelle a été mis sur pied un panel de petites et moyennes entreprises industrielles avec le soutien financier du Conseil régional de Lorraine. Adresse : ERPME, Panel des PMI Lorraines, Groupe ICN, Pôle Lorrain de Gestion, 13, rue M. Ney, 54037 Nancy Cedex, France, tél. : 83396409, télécop. : 83396480. 


\section{RESUMEN}

El objectivo de esta comunicacion es de presentar los resultados de un estudio que se refiere sobre la relacion entre el esfuerzo de investigacion de la empresa y su actividad innovadora en productos. Basados sobre los datos del panel PYME Lorenas, nuestros resultados subrayan la importancia, al nivel del esfuerzo de investigacion, de la dimension recursos humanos y de la orientacion cliente en la discriminacion de las practicas de innovacion producto de la PYME. En cambio, la dimension financiera, asociada tanto a los gastos de investigacion y desarrollo como a los castos de estudios de mercado, no parecen intervenir en la diferenciacion de los comportamientos innovadores.

\section{Introduction}

Les exigences et aspirations nouvelles des consommateurs (Von Hippel, 1982), un environnement technologique en mutation continuelle (Aït-el-hadj, 1989), les impératifs et les pressions concurrentielles de tout ordre, sont autant de défis et d'occasions pour la PME aujourd'hui (Cibert, 1985, 1990).

L'innovation, dénominateur commun de ces enjeux par sa dimension stratégique (Porter, 1985), exige de l'entreprise un comportement de créativité et d'effort de recherche de la nouveauté (Gélinier, 1972). Or, selon la tradition schumpéterienne (Schumpeter, 1951 ; Freeman, 1974), seules les entreprises de grande taille, dotées d'un pouvoir de monopole, sont capables de fournir un effort de recherche (chercheurs, scientifiques, budget $\mathrm{R}$ et $\mathrm{D}$...) nécessaire à la création de produits ou procédés nouveaux. Si cette relation entre effort de recherche et comportement innovatif est toujours controversée (Kamien et Schwartz, 1982), qu'en est-il, plus particulièrement, en ce qui a trait à la PME ?

De manière plus précise, les différentes dimensions de l'effort de recherche sont-elles ou non discriminantes des pratiques de développement et de lancement de nouveaux produits au niveau de la PME ?

L'objet de cette étude est de présenter certains résultats de nos travaux relatifs à cette question, non sans rappeler au préalable le cadre dans lequel ils s'inscrivent.

\section{Cadre de l'étude}

\section{1. Éléments théoriques}

La question de la relation entre l'activité en matière d'innovation et l'effort de recherche, selon la taille de l'entreprise, a fait l'objet de nombreux travaux (Kamien et Schwartz, 1982 ; Rothwell, 1978). Généralement, cette question est 
abordée soit sous l'angle de l'incidence de la taille et du pouvoir de monopole sur l'intensité de l'innovation, soit sous l'angle de l'effet-taille sur l'efficience et l'intensité de l'effort innovatif.

Pour les tenants de l'hypothèse de Schumpeter (1951), la grande entreprise est génératrice de progrès technologiques et source principale du flux des produits nouveaux. En effet, la grande entreprise est la seule à pouvoir gérer et, bien entendu, disposer des moyens matériels, financiers et humains nécessaires à l'innovation. Sa position dominante et la diversification de ses activités lui permettent d'une part d'accumuler des profits considérables, lui assurant ainsi le financement de ses dépenses de $\mathrm{R}$ et $\mathrm{D}$, et d'autre part, de réduire les incertitudes inhérentes à l'activité de recherche (risques d'apparition de produits concurrents et de débouchés limités). Cependant, dans le cadre de ces études, si l'effort de recherche et l'intensité de l'innovation augmentent avec la taille de l'entreprise, un point de retournement pour ce qui est de son efficience est à signaler.

Pour de nombreuses autres études (parmi les plus récentes Pavitt et al., 1987, Acs et Audretsch, 1988, Napolitano, 1991), la relation entre effort de recherche et intensité d'innovation n'est pas aussi claire. La PME, par ses particularités internes (souplesse d'adaptation, flexibilité organisationnelle...) et externes (proximité du marché, écoute des besoins du client...), joue un rôle dans la dynamique de l'innovation que les indicateurs de $\mathrm{R}$ et $\mathrm{D}$, fréquemment retenus, ne laissent pas apparaître (Adriaensens, 1990).

Cette controverse sur la question, posée ici, n'est pas aussi tranchée. Elle laisse transparaître la difficulté que pose la définition de l'innovation (Herrmann, 1991). Dans la perspective schumpéterienne, « le concept d'innovation implique nouveauté, c'est-à-dire rupture qualitative ou quantitative impliquant un saut (non une évolution imperceptible) entre deux états successifs » (Heyvaert, 1973). De fait, cette définition de l'innovation implique un fort potentiel technologique nécessitant dans la plupart des cas une phase de $\mathrm{R}$ et $\mathrm{D}$. Ainsi, les travaux reposant sur cette approche font appel généralement soit au nombre de chercheurs, soit au budget de $\mathrm{R}$ et $\mathrm{D}$ pour mesurer l'effort de recherche, et au nombre de brevets pour saisir l'activité innovatrice.

Dans une autre perspective, l'innovation peut être une simple variation. À l'extrême, on va jusqu'à parler de management de la qualité, du design et plus globalement de «ré-innovation» (Bigoness et Perreault, 1981). Cette stratégie de «ré-innovation» permet, selon Rothwell et Gardiner (1989), «for the manufacturer there were reduced development and testing costs, scale and learning curve benefits, distributed inventories of sparses and servicing experience. For the customer there were familiarity benefits and reduced entry risks...» 
En fin de compte, cette controverse renvoie au débat actuel sur le management du nouveau produit, que Star (1989) qualifie «d'approche marketing plutôt que d'approche technologie».

Dans le cadre de cette étude, nous parlerons d'activité innovatrice en produit dès lors qu'un produit est considéré comme nouveau par l'entreprise qui le développe, même s'il ne s'agit que de modifications apportées à un produit déjà existant (Choffray et Lilien, 1984). Dans la perspective d'une définition de l'innovation, en particulier pour l'activité innovatrice en produit, proche de celle que nous venons de présenter, un certain nombre de travaux récents tiennent compte des diverses dimensions de l'effort de recherche (marketing, $\mathrm{R}$ et $\mathrm{D}$, production...) et de son contexte (structures et stratégies de l'entreprise) dans l'approche intégrée des déterminants du comportement innovatif. Quelques enseignements intéressants sont à souligner sur la relation étudiée

- Une part importante des innovations sont lancées sur les marchés par des entreprises ne faisant état d'aucune dépense de recherche ; ceci laisse apparaître le poids des potentialités non technologiques et des sources non formelles tant internes qu'externes dans le processus innovatif (Pappas, 1984 ; Lunn et Martin, 1986). Par ailleurs, l'interface effort $\mathrm{R}$ et $\mathrm{D}$ effort marketing est intégré au processus d'innovation (pour une synthèse, voir Lilien et Yoon, 1987). Ainsi, en s'intéressant plus particulièrement à l'effet de synergie entre programme $\mathrm{R}$ et $\mathrm{D}$ et recherche marketing, l'entreprise devrait confier la direction de son activité innovative soit à la $\mathrm{R}$ et $\mathrm{D}$, soit au marketing pour optimiser les chances de succès du nouveau produit (Cooper, 1982 ; Gauvin et Lilien, 1989).

- L'innovation n'est pas une finalité en soi. Elle ne peut se comprendre que par rapport à l'histoire, et au présent, de l'entreprise et de son marché. Autrement dit, il n'existe pas un style innovatif, mais une multitude de scénarios stratégiques allant de la position « offensive » à la position « défensive » (Calantone et Cooper, 1981 ; Cooper, 1985). Cette dimension stratégique est déterminante lorsque l'on connaît le poids du dirigeant dans la conduite de la PME (Miller et Toulouse, 1986 ; Khan et Manopichetwattana, 1989).

Dans la lignée de ces travaux, Abeele et Christiaens (1986) concluent, sur la relation effort de recherche-comportement innovatif, que les PME seraient davantage dans une situation de «pression du marché» et la grande entreprise, plutôt dans un contexte de «pression technologique».

Ce survol de la littérature souligne l'intérêt d'autres enquêtes empiriques sur la relation effort de recherche-comportement innovatif, en particulier pour la PME. 


\subsection{Méthodologie}

\subsubsection{La base de données}

Notre analyse empirique a été effectuée sur les données recueillies par nos enquêteurs au cours d'entrevues individuelles auprès des responsables de petites et moyennes entreprises industrielles dans le cadre de la seconde vague d'enquête (1990) du Panel PMI Lorraines ${ }^{1}$. Le tableau 1 présente l'échantillon de travail, constitué de 289 PME industrielles (dont 9 sur 10 ont moins de 200 employés), en fonction de leur activité en matière d'innovation produit et de leurs dépenses de recherche et développement (R et $D)$ et d'études de marché (ETM).

Tableau 1

\section{Présentation de l'échantillon}

\begin{tabular}{|c|c|c|c|c|c|c|}
\hline & DÉPENSE & DE R et D & $\mathrm{T} \mathrm{T}^{-1}$ & & DÉPENS & D'ETM \\
\hline & NON & OUI & & & NON & OUI \\
\hline & & & 13 & & 92 & \\
\hline INNOVE & $(34,07 \%)$ & $(63,55 \%)$ & $R$ et $D(1)$ : & ETM (2): & $(40,00 \%)$ & $(64,41 \%)$ \\
\hline & & & $\begin{array}{l}0,94 \% \\
323,879 \mathrm{FF}\end{array}$ & $\begin{array}{l}0,18 \% \\
61,823 \mathrm{FF}\end{array}$ & & \\
\hline & & & 15 & & 138 & \\
\hline N'INNOVE & $(65,93 \%)$ & $(36,45 \%)$ & R et D: & ETM: & $(60,00 \%)$ & $(35,59 \%)$ \\
\hline & & & $\begin{array}{l}0,45 \% \\
192,755 \mathrm{FF}\end{array}$ & $\begin{array}{l}0,03 \% \\
10,151 \mathrm{FF}\end{array}$ & & \\
\hline & 182 & 107 & 28 & & 230 & \\
\hline TOTAL & $(100 \%)$ & $(100 \%)$ & R et D: & ETM: & $(100 \%)$ & $(100 \%)$ \\
\hline & & & $\begin{array}{l}0,68 \% \\
251,738 \mathrm{FF}\end{array}$ & $\begin{array}{l}0,10 \% \\
33,395 \mathrm{FF}\end{array}$ & & \\
\hline
\end{tabular}

1) Moyenne des dépenses de $R$ et $D$ : en pourcentage du chiffre d'affaires en termes de budget (en francs français)

2) Moyenne des dépenses d'études de marché (ETM) : en pourcentage du chiffre d'affaires en termes de budget (en francs français)

1. Ce panel a été mis sur pied par l'Institut commercial de Nancy (Université de Nancy II) depuis 1988, avec le soutien du Conseil régional de Lorraine. Il fait partie d'un dispositif de recherche européen (Bayad et Herrmann, 1991a).

Les entreprises concernées sont des PMI de 6 à 500 salariés dont l'activité principale relève de la définition de l'industrie au sens strict (y compris les IAA). Sur les 428 PMI répondantes à la première enquête en 1989 (questionnaire portant sur l'exercice 1988), 346 ont accepté à nouveau de recevoir nos enquêteurs pour la seconde vague d'enquête en 1990 (exercice 1989). Cette étude s'appuie sur ces 346 PMI. Mais à cause des valeurs manquantes dues essentiellement aux données financières relatives à l'effort de recherche, l'échantillon final de travail est constitué de 289 PMI lorraines, dont une sur deux a moins de 50 employés (91\% ont moins de 200 employés). 


\subsubsection{Opérationnalisation de la recherche}

Dans le cadre de cette recherche exploratoire (tableau 2), la problématique de la relation entre l'effort de recherche et le développement de produits nouveaux de la PME industrielle fait référence à l'innovation entendue à la fois en tant que processus et résultat du processus (Piatier, 1984 ; Saren, 1984).

TABLEAU 2

\section{Modélisation et opérationnalisation de l'étude}

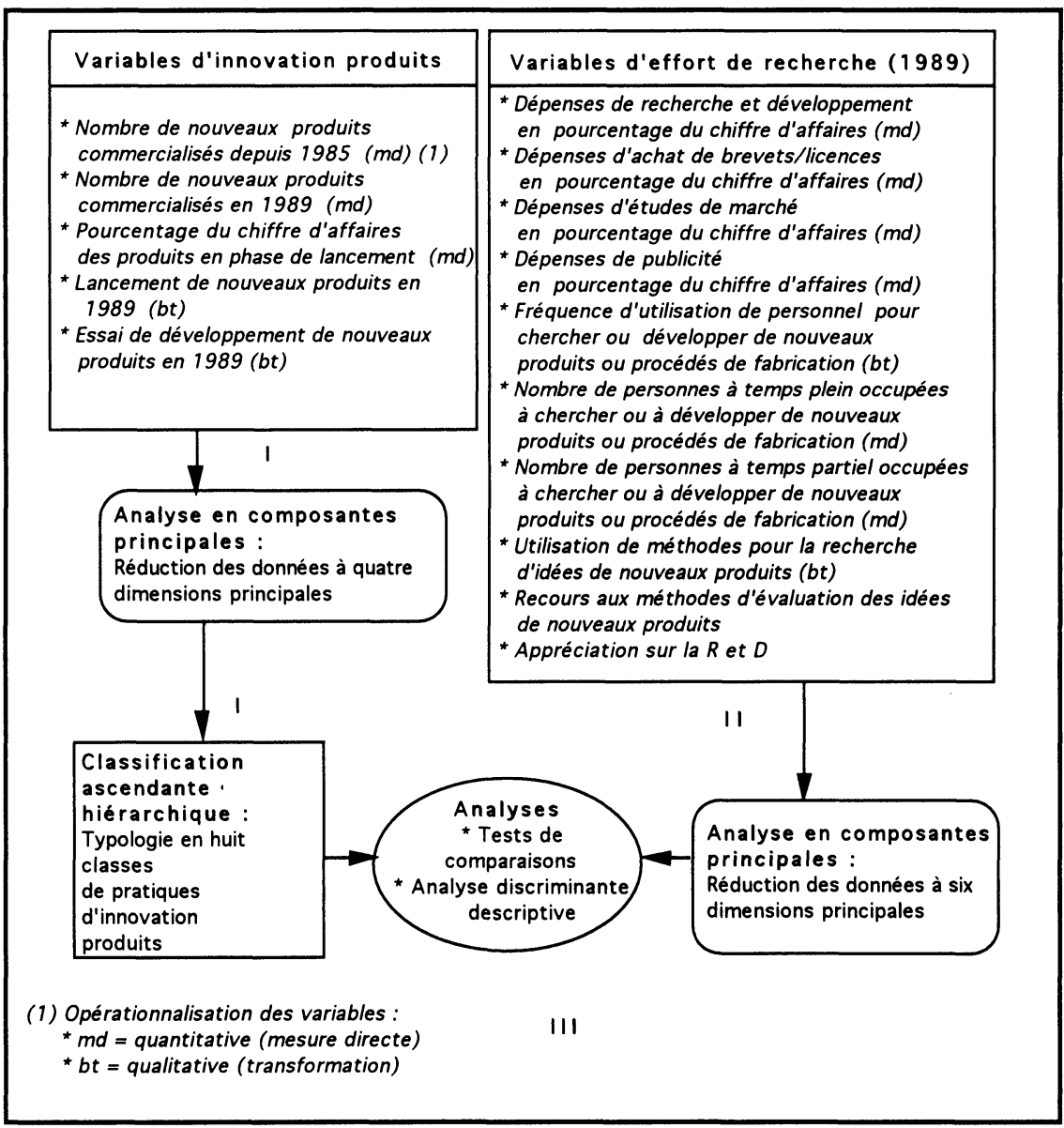

Pour ce qui est du résultat du processus, afin de ne pas alourdir l'analyse, nous n'avons pas fait de distinction selon le type de nouveau produit (original, reformulé, repositionné, voir Gauvin et Lilien, 1989). Nous considérons simple- 
ment qu'un produit est nouveau dès qu'il est perçu comme tel par l'entreprise qui le développe ${ }^{2}$, même s'il ne s'agit que de modifications apportées à un produit déjà existant (Choffray et Lilien, 1984). Cette approche du nouveau produit permet de ne pas éliminer les PME qui innovent sans pour autant faire appel à un contenu technologique fort, voire radicalement nouveau. Ceci est d'autant plus vrai que les innovations absolues (produit et marché) ne représentent qu'une part relativement faible des produits nouveaux commercialisés par les PME/PMI (Brisoux, 1983 ; Bayad et Herrmann, 1991b).

Quant au processus, des auteurs comme Cooper (1983) et Saren (1984) ont déjà souligné que le postulat d'un modèle général était irréaliste étant donné l'extrême variété des processus d'innovation rencontrés dans la réalité (voir aussi Urban et al., 1987). Même si le modèle théorique auquel nous faisons implicitement référence correspond au modèle « standard » du management du produit nouveau (Kotler, 1976, Urban et al., 1987 ; Crawford, 1991), nous insisterons plus sur la nature de l'effort de recherche (processus vu sous l'angle des moyens) que sur sa forme (processus appréhendé sous l'angle séquentiel et hiérarchisé). Ceci permet de substituer à la « vision rationnelle et planifiée de l'innovation » une approche plus intégrative de la dimension informelle de la PME qui est celle de l'entreprise utilisatrice ou sensible à des inputs variés et, probablement, non ordonnés (Saren, 1984).

Étant donné ces quelques remarques conceptuelles, la modélisation et l'opérationnalisation de l'étude (tableau 2) relèvent d'une démarche analytique en trois temps.

Dans un premier temps (I), nous avons dressé une typologie des pratiques en matière d'innovation produit (Cooper, 1979, 1985) en s'appuyant, d'abord, sur une analyse factorielle en composantes principales (dimensions orthogonales) et, ensuite, sur une classification ascendante hiérarchique (méthode de WARD).

Les variables retenues (tableau 2) pour appréhender ces pratiques sont relatives, d'une part, à l'essai de développement de nouveaux produits, ce qui fait davantage référence à la décision stratégique, même non explicite, et d'autre part, au lancement commercial, plus proche du résultat de l'activité innovatrice de l'entreprise (Choffray et Dorey, 1983 ; Habib, 1987). En tenant compte de la définition retenue pour le nouveau produit, l'intensité de cette activité est appréciée par le nombre de nouveaux produits commercialisés en 1989, mais aussi au cours des cinq dernières années (Choffray, 1984 ; Gauvin et Lilien, 1989), et la part du chiffre d'affaires réalisée avec des produits en phase de lancement en 1989.

2. Selon les résultats d'une enquête CEPME-SOFRES de 1981, un dirigeant de PMI sur trois considérait son entreprise comme innovatrice (Cibert et Murat, 1985). Nos propres observations, ainsi que d'autres enquêtes, confirment cet ordre de grandeur. 
La seconde étape de l'analyse (II) consiste à réduire les indicateurs d'effort de recherche, entendue au sens large (tableau 2), à quelques dimensions factorielles principales.

Outre les indicateurs financiers classiques retenus pour saisir l'intensité des efforts de recherche, telles les dépenses de $\mathrm{R}$ et $\mathrm{D}$ et d'études de marché (Gauvin et Lilien, 1989) ou encore les dépenses d'achat de brevets/licences (Kamien et Schwartz, 1982), nous avons inclus dans cette dimension financière les dépenses de publicité comme indicateur de la volonté de communication de l'entreprise (Banoun, 1991). Il faut aussi souligner que les variables d'effort de recherche concernant les moyens humains font davantage référence à la notion de personnel au sens large qu'à la notion de chercheurs au sens R et $D$. En effet, de manière plus spécifique à la PME, nous faisons plutôt référence aux techniciens, aux vendeurs, hommes de production et de terrain impliqués de manière plus ou moins soutenue dans le processus innovatif de l'entreprise (Cooper, 1964). Enfin, nous avons retenu, comme indicateurs plus qualitatifs de l'effort de recherche, des méthodes «moins onéreuses et plus à la portée des petites structures » (Aît-el-hadj, 1989) fondées sur la mobilisation de la créativité et la mise en valeur de l'expérience et des compétences des acteurs (Habib, 1987; Urban et al., 1987).

L'étape finale de l'analyse (III) consiste à mettre en relation les pratiques d'innovation produit et les dimensions principales de l'effort de recherche par des tests de comparaisons et une analyse discriminante.

\section{Résultats}

\subsection{Les pratiques d'innovation produit}

Dans le cadre de la première étape, l'analyse factorielle menée sur les variables d'innovation nous a permis de faire ressortir quatre dimensions représentant $95 \%$ de la variance totale. L'information associée à chacun de ces quatre facteurs principaux ${ }^{3}$ est à la base d'une typologie, en huit classes, des pratiques

3. Premier axe $(33 \%) \longrightarrow$ poids des produits en phase de lancement (ctr $=0,93$ ) et intensité d'innovation en 1989 (ctr = 0,75),

deuxième axe $(25 \%) \longrightarrow$ intensité d'innovation passée (depuis 1985, ctr $=0,96)$ et présente $(1989, \mathrm{ctr}=0,54)$,

troisième axe $(22 \%) \longrightarrow$ lancement de produits nouveaux en 1989 $(\mathrm{ctr}=0,98)$,

quatrième axe $(15 \%) \longrightarrow$ essai de développement de produits nouveaux en 1989 (ctr $=0,99)$. 
TABleaU 3

Profils des classes de la typologie sur les variables d'innovation

\begin{tabular}{|c|c|c|c|c|c|c|c|c|c|c|}
\hline $\begin{array}{l}\text { Typologie } \\
\text { Variables de base }\end{array}$ & $\begin{array}{l}\text { Classe } 1 \\
\text { Inté- } \\
\text { ressées }\end{array}$ & $\begin{array}{c}\text { Classe } 2 \\
\text { Non } \\
\text { innova- } \\
\text { trices }\end{array}$ & $\begin{array}{c}\text { Classe 3 } \\
\text { Jeunes } \\
\text { défensives }\end{array}$ & $\begin{array}{c}\text { Classe } 4 \\
\text { Sélectives }\end{array}$ & $\begin{array}{c}\text { Classe } 5 \\
\text { Flexibles }\end{array}$ & $\begin{array}{c}\text { Classe } 6 \\
\text { Anciennes } \\
\text { offensives }\end{array}$ & $\begin{array}{l}\text { Classe } 7 \\
\text { Techno- } \\
\text { logiques }\end{array}$ & $\begin{array}{c}\text { Classe } 8 \\
\text { Tradi- } \\
\text { tionnelles }\end{array}$ & $\begin{array}{l}\text { Duncan } \\
\text { multiple } \\
\text { range tests } \\
\text { (1) }\end{array}$ & $\begin{array}{c}\text { ANOVA } \\
\text { probabilité } \\
\text { critique } \\
\operatorname{Pr}>\mathbf{F}\end{array}$ \\
\hline $\begin{array}{l}\text { * Nombre } \\
\text { de nouveaux produits } \\
\text { commercialisés } \\
\text { depuis } 1985\end{array}$ & $0(1)$ & 0 & ++ & + & + & ++ & 0 & +++ & 0,05 & 0,0001 \\
\hline $\begin{array}{l}\text { * Nombre } \\
\text { de nouveaux produits } \\
\text { commercialisés en } 1989\end{array}$ & 0 & 0 & + & + & 0 & ++ & ++ & +++ & 0,05 & 0,0001 \\
\hline $\begin{array}{l}\text { * Pourcentage } \\
\text { du chiffre d'affaires } \\
\text { relié aux produits } \\
\text { en phase de lancement }\end{array}$ & 0 & 0 & 0 & + & ++ & + & +++ & ++ & 0,05 & 0,0001 \\
\hline $\begin{array}{l}\text { * Essai de développement } \\
\text { de nouveaux produits } \\
\text { en } 1989\end{array}$ & +++ & 0 & +++ & +++ & +++ & 0 & + & +++ & 0,05 & 0,0001 \\
\hline $\begin{array}{l}\text { * Lancement } \\
\text { de nouveaux produits } \\
\text { en } 1989\end{array}$ & 0 & 0 & +++ & +++ & 0 & +++ & ++ & +++ & 0,05 & 0,0001 \\
\hline
\end{tabular}

1. Les tests de comparaisons de Duncan, au seuil d'erreur de $5 \%$, nous ont permis de noter l'importance relative de chacune des variables de base entre les classes de la typologie : $0=$ pas du tout ou peu élevé, $+=$ moyennement élevé, $++=$ élevé, $+++=$ très élevé. 


\section{TABLEAU 4}

\section{Descriptif des huit classes de la typologie du comportement innovatif}

- Les intéressées (82 entreprises) sont des entreprises qui n'ont pas commercialisé de produits nouveaux depuis 1985, mais elles fournissent toutes un effort de développement en produits nouveaux en 1989 (tableau 2) et en procédés nouveaux. L'intérêt, plus que la nécessité, transparaît dans l'absence de difficultés particulières: croissance de l'activité, position des produits plutôt forte...

- Les non-innovatrices (63 entreprises) se caractérisent surtout par leur petite taille (en général moins de 50 salariés) et leur relative ancienneté (10 à 20 ans). Malgré une position concurrentielle plutôt moyenne et une très faible différenciation des produits, elles se déclarent très peu intéressées tant par l'innovation produit que par l'innovation procédé.

- Les jeunes défensives (30 entreprises) se distinguent par leur faible ancienneté (5 à 10 ans) mais surtout par leur résultat, en général, déficitaire, et par la faiblesse du poids des produits en phase de lancement dans le chiffre d'affaires. On peut aussi noter leurs difficultés de recrutement, en particulier, dans le domaine technique et leur engagement dans la formation à la qualité. L'idée pour le plus important nouveau produit trouve son origine, majoritairement, au sein du département commercial; il s'agit généralement d'un produit modifié.

- Les sélectives (69 entreprises) sont des entreprises, généralement, de taille moyenne (50 à 100 salariés) et très anciennes (plus de 20 ans) qui laissent apparaitre d'une part une certaine modération dans l'intensité d'innovation produit et d'autre part un très fort degré de différenciation de leurs produits. Une des particularités de ces entreprises tient à la diversité des origines de l'idée du principal nouveau produit qui relève tant de la dimension marché que de la dimension technique.

- Les flexibles (8 entreprises) sont, en proportion relative, des moyennes entreprises (plus de 100 salariés). Elles ont une position concurrentielle plutôt forte pour des produits très peu différents. Mais elles connaissent une diminution du rythme de leur activité et s'attendent à un accroissement de la concurrence pour 1992. Malgré une intensité moyenne d'innovation produit depuis 1985 , le poids des produits en phase de lancement dans le chiffre d'affaires demeure relativement élevé. Si elles ont toutes essayé de développer de nouveaux produits, aucune n'en a commercialisé en 1989.

- Les anciennes offensives (15 entreprises) sont de taille moyenne (100 à 200 salariés) et relativement anciennes (10 à 20 ans). Elles se distinguent par leur statut de filiale et le caractère unique de leurs produits. Elles considèrent, cependant, que la position de leurs produits est relativement moyenne face à une concurrence perçue comme très forte. Ce sont aussi des entreprises qui d'une part exportent régulièrement et, d'autre part, n'hésitent pas pour le principal nouveau produit à se lancer parfois sur des marchés totalement nouveaux pour elles. Toujours pour le produit principal, l'idée provient généralement de la maison-mère ou de la comparaison avec les concurrents. La formation professionnelle est d'importance.

- Les technologiques ( 9 entreprises) sont des entreprises très jeunes; près d'une sur deux à moins de cinq ans, les autres ne dépassent pas dix ans d'existence. Elles ont en général moins de 50 salariés. Comme pour les noninnovatrices et les jeunes défensives, le dirigeant est le fondateur de l'entreprise mais à contrario des deux autres groupes, il a généralement moins de 45 ans et possède une importante formation supérieure. Par ailleurs, elles considèrent leurs produits comme très différents avec cependant une position de marché relativement faible. Il faut souligner qu'elles se lancent, pour une bonne part, sur des marchés totalement nouveaux avec le principal nouveau produit en 1989. Ce dernier trouve son origine au sein du département technique ou, dans une moindre mesure, dans une étude de marché.

- Les traditionnelles (13 entreprises) sont en majorité des entreprises de grande taille (200 à 500 salariés) et très anciennes (plus de 20 ans). Le développement et le lancement de nouveaux produits sont soutenus et intenses (tableau 2). Si elles ont des produits uniques et une position de leader, elles perçoivent la concurrence comme forte et prévoient une augmentation de celle-ci pour 1992. Une des particularités de ces entreprises tient à l'âge du dirigeant, il a généralement moins de 45 ans. Il est souvent minoritaire et de formation supérieure. La prise de risque en matière d'innovation produit est cependant limitée, puisque le principal produit commercialisé en 1989 est souvent un produit modifié lancé sur des marchés existants. Les principales sources d'idées pour ce nouveau produit sont le département commercial, les clients et la comparaison avec les concurrents. 
d'innovation produit. Le tableau 3 permet, de manière redondante, de mettre en relief les profils des classes retenues au regard des variables initiales d'innovation.

Parallèlement à cette mise en relief sur les variables d'innovation, l'analyse des groupes est complétée par des tris croisés (tests du Chi deux) sur un ensemble de variables descriptives des comportements et structures de l'entreprise $^{4}$. Ce complément d'analyse, que nous ne présentons pas ici dans le détail, permet de mieux typer les pratiques d'innovation et facilite ainsi la lecture et l'intitulé (tableau 4) de chaque groupe.

\subsection{Les dimensions fortes de l'effort de recherche}

Dans la deuxième étape, les résultats de l'analyse factorielle sur les indicateurs d'effort de recherche nous permettent de faire apparaître une réduction des données en six facteurs principaux qui expliquent $74 \%$ de la variance. Cette structuration en six axes ainsi que les contributions des variables de base à ces axes sont présentées au tableau 5 . La lisibilité des six principales dimensions est facilitée par une rotation Varimax.

Le premier facteur ( $24 \%$ de la variance) est relatif, au premier ordre, à une dimension humaine dans l'effort de recherche; nombre de personnes à temps plein occupées à chercher ou à développer de nouveaux produits ou procédés de fabrication avec une fréquence élevée (toute l'année) d'utilisation de ce personnel. Au second ordre, il semble que ce personnel s'oriente dans sa recherche d'idées, particulièrement vers les études de marchés spécifiques, mais aussi vers les séances de créativité. Toujours au second ordre, on notera le recours à des dépenses d'achats de brevets/licences.

Le deuxième facteur renvoit à une dimension financière associée aux dépenses de Recherche et Développement. L'importance de ces dépenses semble conduire l'entreprise à apprécier la $\mathrm{R}$ et $\mathrm{D}$ comme l'un de ses points forts et à évaluer attentivement ses idées de nouveaux produits.

Le troisième facteur représente une dimension créativité liée à l'utilisation de méthodes pour la recherche d'idées de nouveaux produits; en particulier,

4. Parmi ces variables, les plus significatives sont la taille, la maturité de l'entreprise, la formation du dirigeant, le couple produits/marchés, la croissance de l'activité, la fréquence des exportations, les difficultés de recrutement, la formation, le développement de nouveaux procédés de production, et enfin du type et de l'origine de l'idée pour le produit nouveau dominant. Par contre, le secteur d'activité et les modes de production, par exemple, ne révèlent pas de différences significatives entre les groupes. 


\section{Tableau 5}

\section{Résultats de l'analyse factorielle : après rotation'}

Facteur 1 Facteur 2 Facteur 3 Facteur 4 Facteur 5 Facteur 6

* Dépenses de recherche et déve-

loppement en pourcentage du chiffre d'affaires

$\begin{array}{llllll}-0,034 & 0,735 & -0,135 & -0,240 & 0,037 & -0,332\end{array}$

* Dépenses d'achat de brevets/licences en pourcentage du chiffre d'affaires

$\begin{array}{llllll}0,406 & -0,119 & -0,116 & -0,071 & 0,261 & -0,015 \\ -0,080 & -0,039 & 0,092 & 0,092 & 0,910 & -0,040 \\ & & & & & \\ -0,034 & -0,185 & -0,042 & -0,041 & -0,014 & 0,887 \\ & & & & & \\ 0,730 & -0,216 & 0,109 & 0,508 & 0,045 & 0,041\end{array}$
ou procédés de fabrication

* Nombre de personnes à temps plein occupées à chercher ou à développer de nouveaux produits ou procédés de fabrication

* Nombre de personnes à temps partiel occupées à chercher ou à développer de nouveaux produits ou procédés de fabrication

* Utilisation de méthodes pour la recherche d'idées de nouveaux produits

- Cercles de qualité, groupes de progrès

$\begin{array}{rrrrrr}-0,006 & 0,064 & 0,841 & -0,046 & -0,048 & -0,087 \\ 0,408 & -0,185 & 0,351 & 0,059 & 0,510 & 0,498 \\ & & & & & \\ 0,400 & -0,364 & 0,592 & 0,155 & 0,370 & 0,135\end{array}$

- Séances de créativité, brainstorming

* Recours aux méthodes d'évaluation des idées de nouveaux produits (grille, liste de vérification)

\begin{tabular}{rrrrrr}
$-0,058$ & 0,438 & 0,284 & 0,064 & 0,031 & $-0,038$ \\
$-0,181$ & 0,635 & 0,148 & 0,064 & 0,031 & $-0,038$ \\
$24 \%$ & $35 \%$ & $46 \%$ & $56 \%$ & $65 \%$ & $74 \%$ \\
\hline
\end{tabular}

* Appréciation sur la recherche et développement

Pourcentage cumulé de la variance expliquée

1. Mesure de Kaiser =0,71 (mesure pour l'adéquation de l'échantillonnage). Contributions des variables aux axes factoriels après rotation Varimax. 
les cercles de qualité ou les groupes de progrès. Le recours à des méthodes de créativité est aussi présent. Le quatrième facteur est complémentaire au premier axe puisqu'il est, aussi, associé à une dimension humaine mais cette fois dans une perspective moins intense; nombre de personnes à temps partiel occupées à chercher ou à développer de nouveaux produits ou procédés de fabrication avec une fréquence moyenne (à certains moments de l'année) d'utilisation de ce personnel.

La dimension marché est dominante au niveau du cinquième facteur, notamment à travers les dépenses d'études de marchés. Cette dimension est appuyée qualitativement par l'utilisation d'études de marchés spécifiques comme méthode de recherche d'idées de nouveaux produits.

Enfin, le dernier facteur fait apparaître une dimension client associée aux dépenses de publicité. Outre la communication dans son aspect promotion/image de l'entreprise et de ses produits, cette orientation client est confirmée, au second ordre, par la recherche d'idées liée aux besoins des clients via des études de marchés spécifiques. On notera, toujours parmi les variables de second ordre, l'opposition par rapport aux dépenses de $\mathrm{R}$ et $\mathrm{D}$.

\subsection{Effort de recherche et pratiques d'innovation produit : les tests}

Après avoir synthétisé les données d'un côté, dans une optique classificatoire, et de l'autre, dans une optique factorielle, notre investigation s'est pleinement centrée sur les relations entre effort de recherche et pratiques d'innovation produit. De manière plus précise, les différentes dimensions de l'effort de recherche sont-elles ou non discriminantes des pratiques de développement et de lancement de nouveaux produits?

Les résultats des tests de comparaisons multiples (tableau 6) révèlent qu'au seuil de $5 \%$, seulement trois des six facteurs d'effort de recherche sont significativement reliés aux classes de comportements d'innovation produit.

- Les moyens humains affectés à temps plein (facteur 1) à la recherche (entendue au sens large). Les traditionnelles et les sélectives sont les PMI qui y ont le plus recours.

- Les moyens financiers affectés à la publicité (facteur 6). Les flexibles sont les mieux positionnées en la matière.

- Les moyens humains affectés à temps partiel (facteur 4) à la recherche. 
TABleau 6

Résultats des tests de comparaisons multiples

\begin{tabular}{|c|c|c|c|c|c|c|c|c|c|}
\hline Principaux facteurs & $\begin{array}{l}\text { Classe } 1 \\
\text { Inté- } \\
\text { ressées }\end{array}$ & $\begin{array}{l}\text { Classe } 2 \\
\text { Non } \\
\text { innova- } \\
\text { trices }\end{array}$ & $\begin{array}{l}\text { Classe } 3 \\
\text { Jeunes } \\
\text { défensives }\end{array}$ & $\begin{array}{c}\text { Classe } 4 \\
\text { Sélectives }\end{array}$ & $\begin{array}{l}\text { Classe } 5 \\
\text { Flexibles }\end{array}$ & $\begin{array}{c}\text { Classe } 6 \\
\text { Anciennes } \\
\text { offensives }\end{array}$ & $\begin{array}{l}\text { Classe } 7 \\
\text { Techno- } \\
\text { logiques }\end{array}$ & $\begin{array}{l}\text { Classe } 8 \\
\text { Tradi- } \\
\text { tionnelles }\end{array}$ & $\begin{array}{l}\text { Duncan } \\
\text { multiple } \\
\text { range tests } \\
\text { (1) }\end{array}$ \\
\hline $\begin{aligned} \text { *Facteur 1: } & \text { moyens humains } \\
& \text { affectés à temps-plein } \\
& \text { à la recherche }\end{aligned}$ & $--(2)$ & -- & + & + & -- & -- & - & ++ & 0,01 \\
\hline $\begin{aligned} * \text { Facteur 2: } & \text { moyens financiers } \\
& \text { affectés à la } \mathrm{R} \text { et } \mathrm{D}\end{aligned}$ & 0 & $\cdots$ & 0 & - & + & 0 & ++ & - & ns. tendance \\
\hline $\begin{aligned} \text { *Facteur 3: } & \text { méthodes de créativité } \\
& \text { et d'évaluation des idées }\end{aligned}$ & + & -- & - & 0 & 0 & + & -- & ++ & 0,10 \\
\hline $\begin{aligned} * \text { Facteur 4: } & \text { moyens humains } \\
& \text { affectés à temps partiel } \\
& \text { à la recherche }\end{aligned}$ & + & $\cdots$ & -- & 0 & ++ & - & ++ & + & 0,05 \\
\hline $\begin{array}{cl}* \text { Facteur 5: } & \text { moyens financiers } \\
& \text { affectés aux études } \\
& \text { de marché }\end{array}$ & 0 & - & ++ & + & + & - & - & 0 & ns. tendance \\
\hline $\begin{aligned} * \text { Facteur 6: } & \text { moyens financiers } \\
& \text { affectés à la publicité }\end{aligned}$ & - & -- & + & - & ++ & + & + & 0 & 0,01 \\
\hline
\end{tabular}

1. Seuil de signification des tests de comparaisons de Duncan. Les classes étant déséquilibrées, les tests de Duncan ont été consolidés par des tests $\mathrm{t}$ de Student entre les classes prises deux à deux.

2. --- = pas du tout, $--=$ très faible, $-=$ faible, $0=$ moyen, $+=$ élevé, $++=$ très élevé. 
TABLEAU 7

Résultats de l'analyse discriminante descriptive

\begin{tabular}{|c|c|c|c|c|c|c|c|c|c|}
\hline $\begin{array}{l}\text { Tests univariés } \\
\text { Probabilité critique }\end{array}$ & $\begin{array}{c}\text { Facteur } 1 \\
(0,0001)\end{array}$ & $\begin{array}{c}\text { Facteur } 2 \\
\text { ns }\end{array}$ & $\begin{array}{c}\text { Facteur } 3 \\
\text { ns }\end{array}$ & $\begin{array}{c}\text { Facteur } 4 \\
\text { ns }\end{array}$ & $\underset{\text { ns }}{\text { Facteur } 5}$ & $\begin{array}{c}\text { Facteur } 6 \\
(0,01)\end{array}$ & $\begin{array}{c}\text { Variance } \\
\text { expliquée } \\
(\%)\end{array}$ & $\begin{array}{l}\text { Corrélation } \\
\text { canonique }\end{array}$ & $\begin{array}{l}\text { Probabilité } \\
\text { critique }\end{array}$ \\
\hline Fonction 1 & 0,967 & 0,014 & 0,288 & $-0,023$ & $-0,172$ & $-0,004$ & $73 \%$ & 0,49 & 0,0001 \\
\hline \multirow[t]{2}{*}{ Fonction 2} & $-0,035$ & 0,112 & 0,334 & 0,442 & 0,215 & 0,796 & $17 \%$ & 0,26 & 0,0417 \\
\hline & & & & & & & \multicolumn{3}{|c|}{ Wilk's lambda $=0,332, \mathrm{pr}>\mathrm{F}=0,0001$} \\
\hline
\end{tabular}

Position des moyennes des classes sur les deux axes discriminants

\begin{tabular}{|c|c|c|c|c|c|c|c|}
\hline Traditionnelles & Sélectives & $\begin{array}{l}\text { Jeunes } \\
\text { défensives }\end{array}$ & Technologiques & Flexibles & $\begin{array}{l}\text { Anciennes } \\
\text { offensives }\end{array}$ & Intéressées & $\begin{array}{c}\text { Non } \\
\text { innovatrives }\end{array}$ \\
\hline
\end{tabular}

Fonction 1

\begin{tabular}{|c|c|c|c|c|c|c|c|}
\hline$-0,86$ & $-0,75$ & $-0,64$ & $-0,43$ & $-x, 15$ & 0,19 & ${ }_{0,28}^{x}$ & ${ }_{0,80}^{x}$ \\
\hline Flexibles & Traditionnelles & $\begin{array}{l}\text { Anciennes } \\
\text { offensives }\end{array}$ & $\begin{array}{c}\text { Jeunes } \\
\text { défensives }\end{array}$ & $\begin{array}{l}\text { Techro- } \\
\text { logiques }\end{array}$ & Sélectives & Intéressées & $\begin{array}{c}\text { Non } \\
\text { innovatrices }\end{array}$ \\
\hline
\end{tabular}

Fonction 2

\begin{tabular}{|c|c|c|c|c|c|c|c|}
\hline$-0,69$ & $-0,32$ & $-0,16$ & {$\left[\begin{array}{c}x \\
0,06\end{array}\right.$} & 0,08 & 0,10 & $-x$ & $-x$ \\
\hline
\end{tabular}


Par contre, si les méthodes de créativité et d'évaluation des idées de nouveaux produits sont à la limite de la significativité (seuil de $10 \%$ ), la dimension financière, associée tant aux dépenses de $\mathrm{R}$ et $\mathrm{D}$ qu'aux dépenses d'études de marchés, ne différencie pas fortement les PMI de l'échantillon selon leur activité en matière d'innovation produit.

Outre l'aspect confirmatoire des tests, l'analyse discriminante (tableau 7), au travers des deux principales fonctions canoniques ${ }^{5}$, fait ressortir, de manière plus fine, les deux aspects dominants de l'effort de recherche dans la différenciation des pratiques d'innovation produit pour les PMI de notre échantillon : en premier lieu les moyens humains et, en second lieu, l'orientation client.

\section{Conclusion}

En tenant compte du caractère exploratoire de l'analyse en milieu PMI, et des recherches déjà réalisées dans le domaine du développement et du lancement de nouveaux produits, plusieurs résultats et limites de l'étude sont à souligner.

Comme on pouvait s'y attendre, les pratiques d'innovation produit sont relativement diverses. Les résultats de la typologie sont, à cet égard, révélateurs de scénarios stratégiques variés que l'on retrouve, en tendance, dans d'autres études (Brisoux, 1983 ; Cooper, 1985 ; Khan et Manopichetwattana, 1989). Ainsi, sont tout aussi présentes, mais dans des proportions différentes, les stratégies d'innovation continue mais d'intensité différente (les sélectives et les traditionnelles : $28 \%$ des entreprises de l'échantillon), les stratégies d'innovation discontinue (les flexibles et les jeunes défensives : $13 \%$ ), les stratégies d'innovation offensive (les anciennes offensives et les technologiques: $8 \%$ ), la stratégie d'ouverture à l'innovation (les intéressées : $28 \%$ ) et, enfin, les non-innovateurs $(23 \%)$.

Par ailleurs pour le principal nouveau produit, si pour la PMI la dimension « market-pull » (département commercial, clients, concurrents) est dominante, la dimension « technological-push » (les technologiques) est aussi présente; la combinaison de ces deux dimensions (les sélectives) occupe une place relativement importante.

5. L'analyse discriminante, menée sur les classes de comportement (variable endogène) en fonction des six principaux facteurs d'effort de recherche (variables explicatives), confirme les résultats des tests. La structure canonique totale de la première fonction discriminante ( $73 \%$ de la variance) révèle le poids important de la dimension humaine (facteur 1) dans la séparation des classes d'innovation produit. La deuxième fonction discriminante fait ressortir la dimension client (facteur 6), et dans une moindre mesure, une dimension humaine moins intense (facteur 4 ) et une dimension créativité (facteur 3 ). 
En ce qui concerne à proprement parler la relation effort de recherchecomportement innovatif, les résultats nous incitent à émettre deux séries de remarques :

- L'absence de discrimination forte de la dimension financière de l'effort de recherche (dépenses de $\mathrm{R}$ et $\mathrm{D}$ et d'études de marchés) est à la base de deux implications fortes. La première renvoit à la faiblesse financière structurelle de la PME qui semble être compensée, entre autres, par le savoir-faire (sources internes) et la connaissance du marché (sources externes). Sachant qu'une PMI sur deux innove sans aucune dépense de $\mathrm{R}$ et $\mathrm{D}$, faciliter la gestion de l'innovation de la PME, c'est essayer de comprendre ces moyens non formalisés de recherche pour essayer de réduire "l'anachronisme » du système financier à leur égard (Geoffron, 1991). La seconde implication, en relation avec la précédente, concerne les responsables publics ou financiers. En effet, l'utilisation d'indicateurs financiers, en particulier de $\mathrm{R}$ et $\mathrm{D}$, peut fortement biaiser la mesure de la compétitivité et de l'innovativité de la PME (Napolitano, 1991).

- Le pouvoir discriminant de la dimension humaine et de l'orientation client est à la base des potentialités internes et externes de la PME en matière d'innovation. L'implication qui en découle est qu'une stratégie d'innovation est aussi une stratégie de formation et d'implication de l'homme dans l'entreprise (Meignant, 1991). La gestion de l'innovation est étroitement liée à la gestion des ressources humaines et plus précisément à la gestion des compétences (Morin et Seurat, 1989). En ce qui concerne l'orientation client dans l'effort de recherche, on retrouve en filigrane d'une part le besoin d'une supériorité du produit et d'une adéquation de celui-ci aux attentes des consommateurs et des utilisateurs et, d'autre part, la dimension marketing au travers de l'image, de la promotion et de la qualité du produit.

Les résultats de cette étude doivent cependant être nuancés si l'on garde à l'esprit les limites inhérentes à ce type d'approche que sont les problèmes de mesure de l'activité et du type d'innovation, de la variété du processus innovatif, des indicateurs d'effort de recherche (Gauvin et Lilien, 1989) ; et ceci d'autant plus qu'il s'agit du milieu PME.

En ce qui concerne la mesure du comportement innovatif des entreprises, si ce problème est avant tout d'ordre conceptuel, il convient de souligner que la définition retenue ne permet pas de préciser le degré d'innovation, notamment par rapport au produit, à la technologie et au marché. Cette précision sur le degré innovateur peut conduire à une évaluation plus fine des incidences et particularités de l'effort de recherche de la PME. Tout aussi importante aurait été 
l'introduction du type de marché (demande finale ou dérivée) auquel le nouveau produit est destiné ainsi que la genèse de ce dernier (initiative individuelle, commande spéciale, soumission à un appel d'offre). En effet, ceci ne pourrait qu'améliorer la prise en compte des divers scénarios d'innovation à la lumière de situations fréquemment rencontrées (sous-traitance, marketing industriel...) dans les PME (Brisoux, 1983).

Enfin, les indicateurs d'effort de recherche retenus ne saurait ici prendre entièrement en compte la complexité et l'informel du processus innovatif de la PME. Ainsi, à l'extrême, l'effort de recherche de la PME pourrait ne correspondre qu'à une réponse courante (non planifiée même de manière informelle) ct incertaine à des stimulis internes ou externes (Saren, 1984).

Aussi, et bien que nos résultats demandent à être confirmés, les implications qui en découlent pour les décideurs et les enjeux pour la PME doivent inciter à poursuivre les recherches dans la compréhension du comportement innovatif de cette catégorie d'entreprise.

\section{Bibliographie}

Abeele, P.V. et I. Christiaens (1986), "Strategies of belgian high-tech firms », Industrial Marketing Management, 15, p. 299-308.

ADRIAENSENS, B. (1990), « Réflexions sur les sources non technologiques de l'innovation », Gestion 2000, 4, p. 81-93.

ACS, Z. et B. AUDRETSCH (1988), «Innovation in large and small firms : an empirical analysis », American Economic Review, vol. 78, septembre, p. 688.

AIT-EL-HadJ, S. (1989), L'entreprise face à la mutation technologique, Paris, Éditions d'organisation.

BAYAD, M. et J.L. HeRrmann (1991a), « Panel PMI lorraines : rapport technique de la seconde vague », Centre Régional de Documentation en Gestion, Nancy.

BAyAD, M. et J.L. HERRMANN (1991b), «Bulletin d'information : L'innovation et son financement dans les PMI Lorraines », Centre Régional de Documentation en Gestion, janvier, Nancy.

BENOUN, M. (1991), «Marketing, savoirs et savoir-faire », 2c édition, Paris, Economica.

BigoneSS, W.J. et W.D. PerReault (1981), « A conceptual paradigm and approach for the study of innovations ", Academy of Management Journal, vol. 24, p. 68-82.

BRISOUX, J.E. (1983), "L'innovation dans les petites et moyennes entreprises ", Congrès 1983 de l'ASAC, Université de la Colombie-Britannique. 
Calantone, R. et R.G. CoOPeR (1981), « New product scenarios: prospects for success », Journal of Marketing, vol. 45 (2), p. 48-60.

ChOFFray, J.M. et F. DOREY (1983), «Développement et gestion des produits nouveaux », Paris, McGraw-Hill.

Cibert, A. (1985), « La révolution technologique : un défi et une opportunité », Revue Française de Gestion, juin-juillet-août, p. 106-118.

CiBERT, A. (1990), « Perspectives », Revue Internationale P.M.E., vol. 3 (2), p. 139-145.

Cibert, A. et J. Murat (1985), «Les PMI face aux nouvelles technologies », Revue Française de Gestion, juin-juillet-août, p. 102-106.

COOPER, A.C. (1964), « R\&D is more efficient in small companies », Harvard Business Review, vol. 42 (3), p. 75-83.

COOPER, R.G. (1979), « The dimensions of industrial new product success and failure », Journal of Marketing, vol. 43, p. 93-103.

COOPER, R.G. (1982), « Market-push strategy inhibits industrial, high-tech innovation », Marketing News, 15 (2).

COOPER, R.G. (1985), « Overall corporate strategies for new product programs », Industrial Marketing Management, 14, 1985, p. 179-193.

CRAWFORD, C.M. (1991), « New Products management », 3e édition, Irwin.

Freeman, C. (1974), Economics of Industrial Innovation, Penguin.

GAUVIN, S. et G.L. LILIEN (1989), « Analyse structurelle des déterminants du comportement innovatif des entreprises industrielles », Recherche et Application en Marketing, vol. 4 (2), p. 1-20.

GelinieR, O. (1972), «L'entreprise créatrice », Paris, Éditions Hommes et Techniques.

Geoffron, P. (1991), «Le financement des PME innovatrices : l'exemple américain », Revue Française de Gestion, juin-juillet-août, p. 129-139.

HEYVAERT, H. (1973), «Stratégie et innovation dans l'entreprise », Thèse présentée à l'Université Catholique de Louvain, p. 11.

HerrmanN, J.L. (1991), « À la recherche d'une définition de l'innovation », Communication aux journées doctorales des IAE de l'est, Pôle Lorrain de Gestion, Nancy.

KAMIEN, M.I. et N.L. SchwARTZ (1982), Market Structure and Innovation, Cambridge, Cambridge University Press.

KHAN, A.M. et V. MANOPICHETWATTANA (1989), « Innovative and noninnovative small firms : types and characteristics », Management Science, vol. 35 (5), p. 597-606.

KotLer, P. (1976), Marketing management, Englewood Cliffs, N.J., Prentice-Hall. 
LILIEN, G.L. et E. YOON (1987), « La performance des nouveaux produits industriels: réexamen des recherches empiriques », Recherche et Application en Marketing, vol. 11 (3), p. 53-67.

LUNN, J. et S. MARTIN (1986), «Market structure, firm structure and research and development », Quaterly Review of Economics and Business, 26 (1), p. 31-44.

MeIGnant, A. (1991), Manager la formation, Paris, Éditions Liaisons.

Miller, D. et J.M. Toulouse (1986), «Chief executive personality and corporate strategy and structure in small firm », Management Science, vol. 32 (11), p. $1389-1409$.

MORIN, J. et R. SEURAT (1989), «La gestion des compétences », Harvard-l'Expansion lété, p. 17-24.

NAPOLITANO, G. (1991), «Industrial research and sources of innovation : a crossindustry analysis of manufacturing firms », Research Policy, 20, p. 171-178.

PAPPAS, C. (1984), « Strategic management of technology », Journal of Product Innovation Management, 1 , janvier, p. 30-35.

PAVITT, K. et al. (1987), "The size distribution of innovating firms in the UK : 1945-1983 », The Journal of Industrial Economics, vol. 35, mars.

PIATIER, A. (1984), «L'innovation dans l'industrie : les enseignements de quelques enquêtes », CETEM, mai, Paris.

PORTER, M.E. (1985), « Competitive Advantage », New York, Free Press.

ROTHWELL, R. (1978), « Small and medium sized firms and technological innovation », Management Decisions, 16 (6), p. 362-369.

ROTHWELL, R. et P. GARDINER (1989), « The strategic management of re-innovation », $R$ et $D$ management, 19 (2), p. 147-160.

SCHumpeter, J. (1951), Capitalisme, Socialisme et Démocratie, traduction française, Paris, Payot.

STAR, S.H. (1989), « Comment : marketing versus technology », Sloan Management Review, vol. 31 (1), p. 3.

SAREN, M.A. (1984), «A classification and review of models of the intra-firm innovation process », $R$ et $D$ Management, 14 (1), p. 11-25.

Urban, G.L. et alii (1987), Essentials of New Product Management, Englewood Cliffs, N.J., Prentice-Hall.

VON HIPPEL, E. (1982), «Trouvez vos nouveaux produits chez vos clients », Harvardl'Expansion, automne, p. 62-68. 